\title{
Uso de herramientas profesionales en la docencia universitaria. Una experiencia en ciencias laborales
}

\author{
RICARD CALVO PALOMARES* \\ RAÚL PAYÁ CASTIBLANQUE** \\ ENRIC SIGALAT SIGNES***
}

* Doctor en Ciencias Sociales. Universitat de Valencia, Valencia, España. E-mail: Ricardo.Calvo@uv.es. ORCID: 00oo-0oo30006-4453. Google Scholar: https://scholar.google.es/citations?hl=es\&user=kJwX8l4AAAAJ.

** Doctorando en Ciencias Sociales. Universitat de Valencia, Valencia, España. E-mail: Raul.paya@uv.es. ORCID: 00oo-0oo27967-8660. Google Scholar: https://scholar.google.es/citations?hl=es\&user=zyRyQKgAAAAJ.

*** Doctor en Ciencias Sociales. Universitat de Valencia, Valencia, España. E-mail: Enrique.sigalat@uv.es. ORCID: oooo0001-8146-0532. Google Scholar: https://scholar.google.es/citations?hl=es\&user=BUYVWzYAAAAJ. 


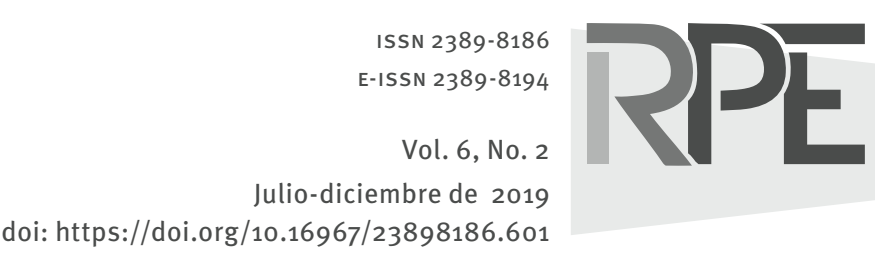

COMO CITAR ESTE ARTÍCULO

How to cite this article:

Calvo, R., Payá, R. y Sigalat, E. (2019). Uso de herramientas profesionales en la docencia universitaria. Una experiencia en ciencias laborales. Revista Perspectiva Empresarial, 6(2), 59-73.

Recibido: 09 de julio de 2019

Aprobado: 02 de octubre de 2019
RESUMEN El artículo presenta la experiencia desarrollada por los autores en los últimos tres cursos académicos en los que han incorporado a su práctica docente el uso de herramientas profesionales con el objetivo de ajustar en mayor medida los contenidos de la materia con la realidad profesional posterior con la que se encontrará el alumnado. Fruto de la suscripción de un acuerdo de colaboración con la Asociación Española de Auditores Socio-Laborales se ha añadido a la docencia -en asignaturas vinculadas con la auditoría sociolaboral- el uso de los formularios, documentos técnicos y el aplicativo informático que los auditores sociolaborales están utilizando en su ejercicio profesional. Tomando como punto de partida esta iniciativa de innovación docente el artículo presenta informaciones sobre la percepción que los alumnos tienen de ella, las principales dificultades con las que se han encontrado y las ventajas que con ello perciben.

PALABRAS CLAVE herramientas profesionales, práctica universitaria, auditoria sociolaboral, utilidad-satisfacción percibida.

\section{Use of professional tools in university teaching. An experience in labor sciences}

ABSTRACT This article presents the experience developed by the authors in the last three academic courses in which they have incorporated the use of professional tools into their teaching practice with the aim of adjusting to a greater extent the subject contents to the professional reality the students will later encounter. As a result, from the signing of a cooperation agreement with the Spanish Association of Socio-Labor Auditors, the use of forms, technical documents and the software application that socio-labor auditors are using in their professional practice has been added to the teaching of subjects linked to socio-labor auditing. Taking this teaching innovation idea as a starting point, the article presents information on the perception the students have of it, the main difficulties they have encountered and the advantages they perceive with it.

KEY WORDS Professional tools, university practice, socio-labor auditing, perceived usefulness-satisfaction. 


\section{Uso de ferramentas profissionais na docência universitária. Uma experiência em ciências laborais}

RESUMO 0 artigo apresenta a experiência desenvolvida pelos autores nos últimos três cursos acadêmicos nos que hão incorporado a sua prática docente o uso de ferramentas profissionais com o objetivo de ajustar em maior medida os conteúdos da matéria com a realidade profissional posterior com a que se encontrará o alunado. Fruto da subscrição de um acordo de colaboração com a Associação Espanhola de Auditores Sócio-Laborais se há agregado à docência -em matérias vinculadas com a auditoria sócio-laboral- o uso dos formulários, documentos técnicos e o aplicativo informático que os auditores sócio-laborais estão utilizando no seu exercício profissional. Tomando como ponto de partida esta iniciativa de inovação docente $o$ artigo apresenta informações sobre a percepção que os alunos têm dela, as principais dificuldades com as que se hão encontrado e as vantagens que com isso percebem.

PALAVRAS-CHAVE ferramentas profissionais, prática universitária, auditoria sóciolaboral, utilidade-satisfação percebida. 


\section{Introducción: si algo ya existe, no hay necesidad de inventarlo}

Intentar acercar la realidad de la docencia de nuestras aulas a la realidad del ejercicio de una determinada actividad profesional es, sin lugar a dudas, uno de los principales retos a los que nos enfrentamos hoy el colectivo de profesores universitarios (Barbosa and Freire, 2019; Mulder et al., 2009). Aunque nuestra misión principal no sea la de crear profesionales estandarizados (Gibbons et al., 1994) tampoco podemos olvidar la generación de espíritu crítico, alternativo y constructivo entre nuestro alumnado; además debemos plantearnos como objetivo la máxima armonización de los contenidos docentes impartidos con la realidad laboral a la que habrán de enfrentarse para mejorar su empleabilidad (Aamodt and Havnes, 2008; McQuaid and Lindsay, 2005; Méhaut and Wich, 2012).

Para ello se considera necesario entrar en contacto con esa realidad profesional y alinearse con los deseos de las empresas (Abraham and Karns, 2009; Martensen and Grønholdt, 2009). Una buena manera es a través de su institución profesional representativa, "colegio oficial" o "asociación profesional homóloga" -en caso de no existir este como tal-, pues es la encargada de mantener la esencia profesional de la actividad (código deontológico); asimismo, asume entre sus finalidades la de construir un mejor futuro para la profesión. Con ese objetivo pone a disposición de sus colegiados y asociados recursos técnicos, formativos, operativos entre otros, que les puedan ser de utilidad para la mejora de su ejercicio profesional.

La experiencia innovadora que presentamos toma estas premisas como básicas, llevándolas a la práctica. Concretamente muestra la iniciativa desarrollada en torno a la auditoría sociolaboral como cuestión de interés central de trabajo y como materia a impartir en las aulas universitarias. Plantea el esfuerzo realizado por vincular el ejercicio profesional con la docencia universitaria y viceversa. Ello ha sido posible merced al acuerdo de colaboración al que llegaron la Asociación Española de Auditores Socio-Laborales -CEALe 'INVESLAB' (Grupo de Innovación en Ciencias Laborales) de la Universitat de València.
En cuanto a la interacción universidadempresa, estudios previos han mostrado la dificultad de establecer redes de cooperación entre la esfera educativa y la productiva. Hall (2003) se refiere a ello como la paradoja de los "dos mundos", derivada de múltiples barreras relacionadas con la orientación (la finalidad de la universidad es desarrollar conocimiento, mientras que las empresas buscan la eficiencia de la innovación) y la transición (burocracias de gestión) en la colaboración entre ambas esferas (Dasgupta and David, 1994). Todo ello supone una menor colaboración entre universidad y empresa que las relaciones entre el propio tejido productivo (clientes y proveedores) (Drejer and Jørgensen, 2005). Derivado de las dificultades identificadas para la colaboración entre ambas instituciones, consideramos relevante los acuerdos de cooperación firmados.

Pese a las barreras de colaboración existentes, son abundantes las investigaciones previas que han hallado una relación positiva en la cooperación empresa y universidad al obtener un mayor potencial de transferencia del conocimiento generado y conseguir innovaciones exitosas para las empresas (Mansfield, 1995; Laursen and Salter, 2004; Petruzzelli, 2011; Belluccia and Pennacchio, 2016; Hewitt-Dundas, Gkypalib and Roperb, 2019). No obstante, menos explorada se encuentra la relación inversa. Es decir los beneficios de la utilización de innovaciones en la esfera productiva en el contexto universitario $\mathrm{y}$ en menor medida sobre las herramientas de auditoría sociolaboral; contexto que pretende explorar la presente investigación.

En el nuevo escenario económico, social y laboral, queda justificada la importancia de acercar posturas y crear puentes de entendimiento y cooperación entre las instituciones de educación superior y el mercado laboral. El centro de actuación se debe situar en el estudiante, el futuro profesional del siglo XXI, pues es él el que debe articular a través de su propio proyecto profesional y vital un conjunto de competencias de acción profesional adquiridas durante la formación superior para incrementar así sus oportunidades en el proceso de transición a la vida activa (Llanes, Figuera y Torrado, 2017; van der Heijde, 2014). Además, tal y como señalan Bozu y Aránega (2017), los futuros profesionales del ámbito educativo demandan una formación más 
integral con un mayor desarrollo de competencias para acceder a un empleo que vaya más allá de la especialización profesional.

Esta iniciativa también pone de manifiesto nuestra apuesta por no caer en el error del que muchas veces adolece el entorno universitario, a saber, de pensar que es el único estamento capaz de generar conocimiento sobre una determinada materia; por esta razón, hemos optado por buscar lo que en el mundo profesional pudiera ya existir y fuera de utilidad para la mejora de la labor docente.

En base a todo esto, el artículo presenta la experiencia desarrollada en los últimos tres cursos docentes en los que se vinculan lo académico con lo profesional. Centra su interés principal en la medición realizada de la percepción que los alumnos tienen, las principales dificultades con las que se han encontrado y las ventajas que con ello perciben. Dada la orientación aplicada de la experiencia el texto se estructura en torno a los aspectos clave de la iniciativa, cómo se ha desarrollado y qué resultados se han obtenido.
Finalmente se dan las conclusiones a modo de líneas de trabajo.

\section{La iniciativa, ¿en qué ha consistido? La necesidad de buscar alianzas estratégicas}

Principalmente la iniciativa se ha concretado en una propuesta de uso académico de las herramientas técnicas utilizadas en el ejercicio profesional como auditores sociolaborales. Tanto para el desarrollo de prácticas puntuales realizadas en el aula (sobre algún aspecto en concreto de la auditoría) como de soporte para la realización del trabajo práctico final planteado, consistente en el desarrollo de una auditoría sociolaboral a una determinada realidad cualquier entidad pública o privada que pudiera ser auditada sociolaboralmente- (tabla 1$)$.

Tabla 1. Resumen de la propuesta docente realizada

Objetivo

Realización de prácticas puntuales

sobre contenidos en concreto del

temario de la asignatura
Aspecto profesional utilizado

Documentos de soporte del proceso de auditoría: carta de encargo o contrato de auditoría; presupuesto; plan de auditoría; checklist o listas de indicadores; carta de manifestaciones o informe final 
Antes de avanzar, cabe mencionar que la auditoría sociolaboral se define como toda evaluación sistemática (proceso lógico y secuencial) de hechos fehacientes documentables (periódicos en el tiempo) realizados por un profesional con objetividad, independencia y neutralidad (Torras y Ribas, 2005) sobre la gestión y políticas de recursos humanos, el cumplimiento de las obligaciones legales en materia laboral y contable con objeto de proporcionar acciones de mejora (Fuentes, Morales, Veroz, 2005). Por lo que se puede extraer de dicha definición, existen tres tipos de auditoría sociolaboral según su contenido y tal como manifiestan Campamá (2006) y Ciruela (2009) pueden dividirse en: (i) auditorías de legalidad, de comprobación o de administración, dedicas a comprobar si la organización cumple con las obligaciones en materia de derechos (al trabajo, seguridad social, prevención de riesgos laborales entre otros); (ii) auditorías de eficacia, de gestión u operativa, que van más allá del mero cumplimiento legal, centradas en la función de recursos humanos (descripción de puestos de trabajo, reclutamiento y selección, formación y capacitación entre otros) y (iii) auditorías de eficacia, de gestión u operativa, centradas en la alineación de la dirección estratégica de recursos humanos con la estrategia competitiva de la organización.

Como podemos ver en la tabla 1 , el acuerdo de colaboración existente entre la Universitat de València (a través del grupo INVESLAB) y la CEAL ha permitido el uso académico de su aplicativo informático llamado CEAL Pro. Asimismo, del uso de documentos técnicos 'normalizados' para el desarrollo de una auditoría sociolaboral conforme a la metodología utilizada por la CEAL. Carta de encargo, presupuesto, plan de auditoría, checklist e indicadores o informe final de auditoría, son algunos de los más destacados.

Cabe recordar que, en septiembre de 1993, el Consejo General de Colegios Profesionales de Graduados Sociales (Licenciados en Relaciones
Laborales y Recursos Humanos) de España impulsó y promovió la creación del Centro Europeo de Auditores Socio-Laborales -CEAL- ${ }^{1}$ como asociación interprofesional independiente de ámbito estatal; con el objeto de fomentar, desarrollar, regular y ordenar la actividad del auditor sociolaboral. Lo que constituye una de las principales actividades profesionales reconocidas a los graduados sociales.

Desde 2014, la colaboración de la Universitat de València y la CEAL ha sido cada vez mayor desde el nombramiento del profesor universitario Ricard Calvo como asesor científico de la asociación ${ }^{2}$.

Esta relación de más de cinco años ha permitido generar multitud de beneficios para ambas partes. El más básico ha sido el de consolidar un vínculo de intercambio permanente entre el estamento profesional y el académico. No podemos obviar la idea de que la universidad es la entidad que reconoce en último término el ejercicio profesional, siendo requisito necesario en cualquier proceso de configuración profesional y sin el que una actividad que opta a ser reconocida como profesión difícilmente lo conseguirá. Este intercambio ha permitido la consolidación de un lenguaje común al referirse a la auditoría sociolaboral, normalización técnica que era una clara resistencia en su proceso de implantación y uso. No podemos dejar tampoco de lado que la universidad es la encargada de determinar si una materia puede llegar a convertirse en un asunto de interés científico. En este sentidola colaboraciónha conseguido abrir una nueva línea de investigación específica sobre la materia, posibilitando el estudio de su realidad y potencialidades futuras al ir apareciendo trabajos de fin de grado y de máster sobre algún aspecto vinculado con la auditoría sociolaboral. Ha permitido, por un lado, que los profesionales visiten la universidad y aporten su visión aplicada (por ejemplo, en actividades complementarias de la titulación o en jornadas de

1 Con Registro Nacional de Asociaciones del Ministerio del Interior, con el número 132722, pasó en 2005 a llamarse Asociación Española de Auditores Socio-Laborales (manteniendo el acrónimo anterior de CEAL).

2 El paso de los años ha permitido que también se convirtiera en miembro de su Comisión Técnica de Formación, incluido en los Comités Organizadores de los Congresos Nacionales y Jornadas Técnicas propuestas, coautor en la publicación de manuales sobre la materia o partícipe en proyectos conjuntos de investigación. 
carácter científico organizadas al efecto) ${ }^{3} ; \mathrm{y}$, por otro, que el profesorado universitario participe en los congresos y jornadas técnicas organizadas desde la asociación ${ }^{4}$.

Lo anterior, ha permitido desarrollar proyectos comunes de interés mutuo tanto a nivel de publicación de manuales y artículos sobre la materia que permiten dar a conocer su potencial ${ }^{5}$ como de investigaciones y estudios conjuntos relacionados que permitan su transferencia a la sociedad (en este caso, a lo sociolaboral) y sea una realidad. Labor de difusión social de gran utilidad y sin la que nuevas actividades laborales no llegan a ser conocidas ni implementadas. En este sentido destacamos dos estudios realizados en 2013-2014 sobre la "Realidad de la auditoría sociolaboral en España. Un balance de situación" y en 2015-2016 sobre "Nuevas posibilidades de negocio para los despachos laborales iel momento de incorporar un auditor sociolaboral?", en colaboración también con el Colegio Oficial de Graduados Sociales de Valencia -COGRASOVA-.

Con todo esto, uno de los grandes beneficios -y el que se convierte en la razón de ser de este artículo- es el de mejorar el cumplimiento de los objetivos docentes propuestos. Permitiendo un mejor ajuste de la oferta académica a la realidad de la profesión. Aspectos que se presentan a continuación.

\section{Método}

\section{Objetivos}

A partir de la experiencia iniciada de manera progresiva desde hace tres cursos tanto en grado, posgrado como títulos propios, nos proponemos conocer la percepción que el alumnado tiene de esta propuesta docente. Por eso, los objetivos que nos planteamos fueron:

OB1: conocer cómo los alumnos perciben la utilidad de incorporar en la práctica docente el uso de herramientas técnicas de carácter profesional.

OB2: conocer el grado de satisfacción de los alumnos sobre los métodos, prácticas y contenidos de la herramienta utilizada; además, conocer la relación entre las dimensiones de satisfacción con la percepción de utilidad.

OB3: identificar los aspectos positivos y negativos de la utilización de la herramienta profesional en las aulas.

\section{Muestra}

En la presente investigación han participado 131 alumnos de los últimos dos cursos académicos de la asignatura "Técnicas de auditoría sociolaboral" del 3er curso del Grado de Recursos Humanos y Relaciones Laborales de la Universitat de València (dos grupos por curso) (tabla 2). Asignatura troncal de carácter obligatorio con una carga docente de 6 créditos del European Credit Transfer and Accumulation System -ECTS-, que se imparte en el primer cuatrimestre y cuyo objetivo básico es la formación de los estudiantes en el conocimiento operativo de los diferentes métodos y técnicas para la auditoría del sistema de relaciones laborales y recursos humanos.

\footnotetext{
3 Todos los años algún representante de la CEAL participa en alguna actividad complementaria en la que se presenta la asociación y su aplicación informática. A la vez han participado en diversas ediciones de las Jornadas sobre Investigación Sociolaboral de la Comunitat Valenciana, que se realizan anualmente en la Facultad de Ciencias Sociales.

4 En este sentido Ricard Calvo ha sido ponente en los últimos 4 Congresos Nacionales sobre Auditoría Sociolaboral celebrados en Madrid (2013), Málaga (2015), Valencia (2017) y Sevilla (2019).

5 Valgan como ejemplos artículos publicados en 2015 y 2016, así como el manual: Auditoría sociolaboral. Teoría y práctica de una herramienta para la gestión de los RRHH, publicado por la editorial Tirant Lo Blanch.
} 
Tabla 2. Muestra del estudio

\begin{tabular}{cccccc} 
Curso & Grupo & $\begin{array}{c}\text { Matriculados } \\
\text { (N) }\end{array}$ & Media asistencia & Respuestas obtenidas (n) & $\begin{array}{c}\text { \% de respuesta } \\
\text { obtenida } \mathbf{a}^{\mathbf{a}}\end{array}$ \\
\hline \hline $2017-2018$ & A & 46 & 39,2 & 35 & 89,3 \\
& B & 43 & 39,6 & 32 & 70,3 \\
\hline \hline $2018-2019$ & A & 52 & 38,4 & 27 & 82,0 \\
\hline \hline Total & B & 57 & 45,1 & 37 & $\mathbf{8 0 , 7}$ \\
\hline \hline
\end{tabular}

Nota: a calculado respecto de la media de asistencia.

Fuente: elaboración propia por parte de los autores.

\section{Instrumento}

El estudio fue diseñado con objeto de realizar una triangulación metodológica, que integre tanto un enfoque cuantitativo como cualitativo, para obtener datos en profundidad y dar validez a la investigación (Johnson, Onwuegbuzie and Turner, 2007). Los dos primeros objetivos fueron estudiados desde un enfoque cuantitativo, mientras que el tercero fue recogido a través percepciones cualitativas de los alumnos. Para ello se ha utilizado un cuestionario administrado a través del aula virtual de la Universitat de València que contenía 12 preguntas; 10 de las cuales fueron escalares y que permitieron la obtención de una valoración numérica (la escala utilizada fue de 0-10 en todas las cuestiones), así como recoger las aportaciones de carácter cualitativo en forma de citas textuales de los comentarios aportados sobre dos preguntas abiertas en las que se solicitó al alumno que señalara los tres aspectos más positivos y negativos de la herramienta profesional. No obstante, para el desarrollo de la presente investigación, nos centramos en analizar 7 de las 12 preguntas del cuestionario (tabla 3).

Tabla 3. Dimensiones y preguntas del cuestionario

Dimensión Preguntas

Utilidad práctica

1. ¿En qué medida consideras útil el ejercicio realizado para la práctica profesional?

(escalar)

Satisfacción (escalar)
2. ¿Cuál es tu grado de satisfacción con la metodología utilizada?

3. ¿Cuál es tu grado de satisfacción con las prácticas realizadas?

4. ¿Cuál es tu grado de satisfacción con los contenidos impartidos?

5. ¿Cuál es tu grado de satisfacción con los materiales de soporte utilizados?

Preguntas abiertas

6. Enumera cuáles han sido los 3 aspectos que más te han gustado.

7. Enumera cuáles han sido los 3 aspectos que menos te han gustado.

Fuente: elaboración propia por parte de los autores. 


\section{Análisis de datos}

Para el análisis de las informaciones recogidas, en primer lugar, se optó por realizar un análisis descriptivo a través del cálculo de las puntuaciones medias y las desviaciones estándar de las cinco variables cuantitativas utilizadas y así poder abordar el primer objetivo y parte del segundo (es decir, conocer la percepción del alumnado sobre el grado de utilidad de la herramienta profesional y su nivel de satisfacción con el ejercicio práctico realizado en las aulas). Asimismo, se calculó el Alfa de Cronbach para medir la consistencia interna de las cuatro variables (metodología utilizada, prácticas realizadas, contenidos impartidos $\mathrm{y}$ soportes utilizados) que miden la satisfacción. Se consideró que la consistencia interna de la escala de medida del Alfa de Cronbach fue adecuada (George and Mallery, 2003) y valida (Huh, Delorme and Reid, 2016) cuando el resultante oscilo entre 0,7 a 0,8 .

En segundo lugar se utilizó la correlación de orden cero para explorar las relaciones existentes entre la variable dependiente (utilidad práctica) y las independientes de satisfacción (métodos, prácticas, contenidos y materiales de soporte utilizados) a través del cálculo del coeficiente $\mathrm{r}$ de Pearson con un nivel de confianza del 95 $\%$; entendiendo que existía una asociación estadísticamente significativa entre las variables con un $\mathrm{p}$ valor $=<0,05$. En tercer lugar, para finalizar con el análisis del segundo objetivo, se utilizó una regresión lineal múltiple para determinar la influencia relativa de cada una de las cuatro dimensiones de participación sobre la utilidad práctica de la herramienta profesional. En el análisis de los datos utilizamos el programa estadístico SPSS (versión 24) que permitió un tratamiento estadístico adecuado.

Finalmente se completó el estudio a través del análisis del discurso de las preguntas abiertas planteadas para abordar el último de los objetivos, solicitando al alumnado que enumerara los aspectos positivos y negativos del ejercicio práctico profesional planteado, permitiendo obtener sus percepciones e identificar relaciones con los niveles de utilidad y satisfacción hallados en los análisis estadísticos y establecer acciones de mejora para los próximos cursos académicos.

\section{Resultados}

\section{Resultados descriptivos}

Los resultados del análisis descriptivo mostraron una percepción positiva de los alumnos sobre la herramienta CEAL Pro tanto por su utilidad práctica (media=6,43; DT=1,65) como por la satisfacción en su realización, siendo la propia práctica profesional realizada la que proporcionó un mayor grado de satisfacción entre el alumnado con una media de 6,66 (DT=1,79) y un tanto menor sobre los métodos, contenidos y materiales utilizados con puntuaciones medias que oscilaron desde el 6,32 (DT=1,67) (para los métodos) hasta el 6,09 (DT=1,49) (sobre los materiales de soporte utilizados) (tabla 4). Así que podemos afirmar que los resultados obtenidos fueron moderados dado que los valores oscilaron en torno a un 6 sobre 10 , poniendo de manifiesto que todavía hay un espacio considerable para la mejora. 
Tabla 4. Análisis descriptivo y confiabilidad

\begin{tabular}{llll}
\multicolumn{1}{c}{ Dimensión } & Preguntas & Media & DT \\
\hline \hline \multirow{2}{*}{ Utilidad } & Valoración global de la utilidad práctica & 6,43 & 1,65 \\
\hline \hline \multirow{2}{*}{ Satisfacción } & Metodología utilizada & 6,32 & 1,67 \\
& Prácticas realizadas & 6,66 & 1,79 \\
& Contenidos impartidos & 6,20 & 1,55 \\
& Materiales de soporte utilizados & 6,09 & 1,49 \\
\hline \hline
\end{tabular}

Nota: 311 alumnos participantes; DT= desviación típica.

Fuente: elaboración propia por parte de los autores.

\section{Análisis de correlación}

En cuanto al análisis de correlaciones, los resultados mostraron una asociación positiva elevada y estadísticamente significativa entre la dimensión de la valoración de la utilidad de la herramienta práctica y las cuatro variables vinculadas con la satisfacción (figura 1). Se identificó una correlación positiva entre la utilidad y la satisfacción en la metodología utilizada $(\mathrm{r}=0,939 ; \mathrm{p}=0,000)$, así como con la actividad práctica realizada $(\mathrm{r}=0,917 ; \mathrm{p}=0,000)$, los contenidos impartidos ( $\mathrm{r}=0,956 ; \mathrm{p}=0,000)$ y los materiales de soporte utilizados $(r=0,914$; $\mathrm{p}=0,000)$.

Así pues, todas las variables que midieron el grado de satisfacción presentaron una relación bilateral positiva (valores de $r$ cercanos a 1) y estadísticamente significativa (todos los valores fueron de $\alpha$ de 0,000 por debajo del $p=<0,05$ ) con la percepción de la utilidad práctica. Por lo tanto se consideró oportuno incluir las cuatro variables de satisfacción en el análisis de regresión múltiple derivado, ya que todas presentaron una capacidad explicativa elevada sobre la utilidad percibida por el alumnado. Además se observó relaciones positivas entre las propias variables de satisfacción, confirmando los resultados obtenidos con el cálculo del Alfa de Cronbach, en la medida en que los métodos utilizados se correlacionaron con las prácticas realizadas $(\mathrm{r}=0,913 ; \mathrm{p}=0,000)$, los contenidos $(r=0,894 ; p=0,000)$ y los materiales de soporte $(\mathrm{r}=0,855 ; \mathrm{p}=0,000)$; así como las prácticas con los contenidos $(\mathrm{r}=0,834 ; \mathrm{p}=0,000) \mathrm{y}$ los materiales ( $\mathrm{r}=0,790 ; \mathrm{p}=0,000)$, y estos últimos con los propios contenidos de la práctica $(\mathrm{r}=0,934$; $p=0,000$ ).

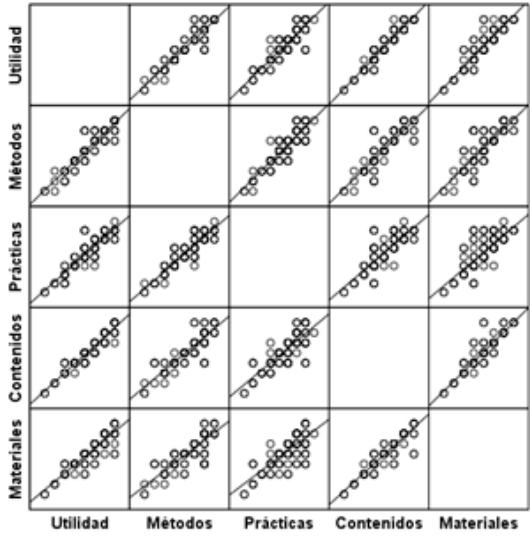

Figura 1. Correlación entre las variables. Fuente: elaboración propia por parte de los autores. 


\section{Análisis de regresión múltiple}

Por último, el análisis de regresión múltiple permitió identificar cuál de las cuatro variables de satisfacción del alumnado (métodos, prácticas, contenidos y materiales) explicó con mayor fortaleza la utilidad percibida por dichos alumnos.

En primer lugar los resultados que se obtuvieron en el modelo (tabla 5) mostraron como las variables predictoras de satisfacción explicaron el 96,4\% de la varianza total (r2= 0,964; p=0,000); es decir la satisfacción del alumnado vendría a explicar prácticamente la totalidad de la percepción de los mismos sobre la utilidad de la herramienta CEAL Pro en la práctica universitaria, dejando tan solo un 3,6 \% de casos atribuibles a otros factores no ponderados en la presente investigación.

Tabla 5. Coeficiente de regresión para la percepción global de utilidad

\begin{tabular}{lcccc} 
Variables & B & B & T & P \\
\hline \hline Constante general &,- 203 & & $-1,720$ &,- 088 \\
Metodología utilizada &,- 152 &,- 154 & 3,033 &,- 003 \\
Prácticas realizadas & 0,281 &,- 305 & 7,434 & -.000 \\
Contenidos impartidos & 0,487 &,- 456 & 8,410 &,- 000 \\
Materiales de soporte utilizados & 0,129 &,- 116 & 2,483 & 0,014 \\
& & & & \\
\hline \hline
\end{tabular}

Fuente: elaboración propia por parte de los autores.

En segundo lugar cabría apuntar que las dos dimensiones que con más fuerza explicaron la percepción de utilidad general del aplicativo informático CEAL Pro fueron: (i) los contenidos formativos impartidos, aspecto que vendría a alcanzar un coeficiente de regresión estandarizado de $(\beta=0,456)$ y que explica un $45,6 \%$ de los casos; (ii) el elemento práctico del ejercicio final propuesto, siendo predictivo del 30,5\% la utilidad global $(\beta=0,305)$ y que explica entre ambos factores de satisfacción un porcentaje elevado del modelo global sobre la utilidad percibida del alumnado. cualitativo. Los aspectos cualitativos recogidos en las preguntas abiertas del cuestionario mostraron una cierta relación con análisis cuantitativos realizados en la medida en que las puntuaciones medias obtenidas identificaron un impacto positivo moderado (alrededor de 6 sobre 10), denotando la existencia de aspectos positivos y otros mejorables que la perspectiva cualitativa nos permite analizar. Lo vemos con mayor claridad en la siguiente tabla donde recogemos dichas aportaciones ordenadas por grado de repetición (tabla 6).

\section{Análisis cualitativo (aspectos positivos y negativos)}

Como ya hemos expuesto, además de la dimensión cuantitativa, también nos propusimos obtener algunas informaciones de carácter 
Tabla 6. Resumen aportaciones cualitativas (ordenadas por grado de repetición)

\begin{tabular}{ll}
\multicolumn{1}{c}{ Ventajas } & \multicolumn{1}{c}{ Dificultades } \\
\hline \hline 1. Genera aprendizaje & 1. Mucho trabajo \\
2. Es útil para la formación del alumnado & 2. Dificultad mayor \\
3. Refleja la realidad profesional de la actividad & 3. Exigencia (requiere esfuerzo extra) \\
& 4. Comparación con el trabajo realizado en otros grupos \\
& 5. Son cosas nuevas, se sale de lo habitual \\
\hline \hline
\end{tabular}

Fuente: elaboración propia por parte de los autores.

Como pondremos de manifiesto en la discusión posterior, destacamos que los alumnos consideraron que el ejercicio práctico fue útil para su aprendizaje y que sus contenidos han permitido acercarlos a la práctica habitual de la auditoría sociolaboral. No obstante, también se recogieron algunas dificultades en torno al volumen de trabajo que suponía su realización y a la dificultad en el manejo de documentación de carácter profesional derivado de su novedad y desconocimiento. Esto queda de manifiesto en los siguientes comentarios textuales que reproducimos en la tabla 7 .

Tabla 7. Resumen de aportaciones cualitativas (citas y comentarios textuales)

\begin{tabular}{lc}
\multicolumn{1}{c}{ Ventajas } & \multicolumn{1}{c}{ Dificultades } \\
\hline \hline "hemos aprendido mucho..." & "hemos aprendido, pero caray nos ha costado..." \\
"el objetivo estaba claro..." & "la exigencia ha sido muy alta, en otros grupos lo han \\
"más asignaturas deberían apostar por esta forma de & tenido más fácil..." \\
trabajar en el aula..." & "cuando nos planteó el trabajo final, nos asustó, no \\
"es una posibilidad muy interesante..." & sabíamos por dónde empezar..." \\
"en el fondo ha sido bueno para nosotras..." & "demasiado trabajo para un cuatrimestre..." \\
"currar, sí mucho, pero aprendiendo..." & "no me parece justo, los otros grupos no han hecho ni la \\
"salimos de la asignatura con una visión más real de lo & mitad que nosotros y han sacado más nota..." \\
que hacen los auditores..." & "buff, hemos sudado la gota gorda..." \\
"por fin una asignatura útil, la mayoría no pasan de la & "muy exigente..." \\
teoría, demasiado teóricas..." & "la idea es buena, pero..." \\
\hline \hline
\end{tabular}

Fuente: elaboración propia por parte de los autores. 


\section{Discusión y conclusiones}

Con la intención de contribuir en la mejora del conocimiento de las competencias que se desarrollan para favorecer los procesos de inserción sociolaboral desde la formación universitaria la presente investigación ha profundizado en esta dirección, deteniéndose en las percepciones y valoraciones del alumnado del Grado de Relaciones Laborales y Recursos Humanos ante la implantación de la propuesta innovadora del uso de herramientas profesionales en su proceso de aprendizaje. Como ya hemos expuesto, con ello se pretendía la mejora de las competencias aplicadas del alumnado por medio de un ajuste entre la visión aportada a través de la academia con la visión que el campo profesional está aplicando.

La investigación presentada puede ser el punto de partida para abrir una reflexión pedagógica sobre el tema del uso de herramientas técnicoprofesionales en espacios universitarios y de la consiguiente búsqueda de alianzas estratégicas de carácter académico-profesional tanto con empresas especializadas como con asociaciones o colegios profesionales.

La experiencia llevada a cabo ha permitido proponer una mejora docente en una especialidad -las ciencias laborales- que no ha sido prioridad tradicionalmente ni entre la oferta docente ni como interés en el proceso innovador. Esperamos que con este tipo de aportaciones también consigamos con el tiempo posicionar mejor este tipo de estudios que tanta importancia están adquiriendo en la sociedad.

En este sentido los resultados obtenidos sobre el primer y segundo objetivo de la investigación ponen de manifiesto una clara tendencia común entre los estudiantes encuestados sobre la utilidad que perciben y su satisfacción con la iniciativa docente propuesta. Aspecto que consideramos de gran interés. Lo vemos con claridad en la tabla 4 en los valores otorgados a las puntuaciones medias donde los alumnos han puntuado con un 6,43 sobre 10 a la utilidad de la herramienta, a las dimensiones de satisfacción con un 6,09 y un 6,66 para los materiales de soporte utilizados y la práctica realizada en el aula respectivamente. También se observa en el rango de respuestas otorgado -concretamente en su desviación típica- a las variables cuestionadas en la que la máxima en la dimensión prácticas realizadas fue del 1,79; esto indica la centralidad de las puntuaciones y a la vez valida nuestra apuesta docente. Pese a ello, y como hemos mencionado, los promedios reflejan unos valores que todavía permiten detectar un espacio considerable para la mejora futura.

En cuanto a las relaciones de ambas dimensiones (es decir, entre utilidad y satisfacción), referentes al segundo de los objetivos de la investigación, los análisis de correlación (figura 1) han permitido constatar una elevada asociación entre ellas; así pues, aquellos alumnos más satisfechos fueron los que percibieron más útil la herramienta y a la inversa. Además, cabe mencionar que dentro de las dimensiones de satisfacción el propio contenido documental fue el que más predecía la utilidad de la herramienta sociolaboral.

En referencia al tercer objetivo las dificultades percibidas han girado en ambos cursos en torno a que esta nueva apuesta docente genera una mayor exigencia para el alumnado, así como una mayor dedicación y carga de trabajo para poder superar la asignatura. Esto queda de manifiesto con mucha claridad en la mayor parte de las aportaciones que los encuestados hacen en las preguntas abiertas sobre ventajas y desventajas de la metodología aplicada (tabla 7).

Una de las limitaciones del estudio puede apreciarse en que la muestra con la que se trabaja procede únicamente de alumnado de grado, siendo necesario para un futuro ampliar esta población de referencia a otros niveles en los que ya se está aplicando la iniciativa.

Teniendo en cuenta todas estas aportaciones, además de las líneas de prospección y limitaciones del estudio, queda patente que la formación inicial -académica- de los futuros egresados universitarios debe continuar en la línea propuesta por el Espacio Europeo de Educación Superior -EEES- a través de una mejora del proceso enseñanza-aprendizaje que aporte realmente las competencias esenciales para una adecuada transición a la vida activa de los universitarios. 
Asimismo, los resultados han evidenciado la necesidad de seguir midiendo para observar si la situación mejora o se estanca. Se trata de un indicador continuo en el tiempo. De igual manera se debe ampliar esta medición a otros niveles educativos, más allá de donde está siendo aplicada la experiencia. La evaluación de la eficacia de esta iniciativa todavía se tiene que profundizar con la ampliación del número de contextos experimentales y con el análisis de los factores que influyen sobre posibles diferentes resultados. Es nuestra intención ampliar en los próximos cursos la medición de las percepciones, utilidades y satisfacción del alumnado con esta propuesta metodológica al resto de contextos propuestos. También está previsto incorporar en futuras investigaciones medidas objetivas para el rendimiento académico, la evaluación de las prácticas por tutores de empresa, la facilidad para encontrar empleo e integrarse laboralmente y el interés que puede despertar está salida profesional en los estudiantes.

Con todo esto, entendemos que los resultados obtenidos validan la apuesta innovadora de buscar una aproximación de lo profesional con lo académico. En cualquier caso, es importante buscar sinergias de intercambio y cooperación entre el contexto profesional y el académico para encontrar el equilibrio y transferencia que exige el EEES. Lo que implica seguir trabajando y fortaleciendo estas relaciones académicoprofesionales en las que ambas partes salen beneficiadas.

\section{Referencias}

Aamodt, P.O. and Havnes, A. (2008). Factors affecting professional job mastery: quality of study or work experience? Quality in Higher Education, 14(3), 233-248.

Abraham, S.E. and Karns, L.A. (2009). Do business schools value the competencies that businesses value? Journal of Education for Business, 84(6), 350-356.

Barbosa, I. and Freire, C. (2019). Portuguese employer's perceptions on management undergraduates' transferable competencies.
Journal of Management Development, 38(2), 141-156.

Bellucci, A. and Pennacchio, L. (2016). University knowledge and firm innovation: evidence from European countries. The Journal of Technology Transfer, 41(4), 730-752.

Bozu, Z. y Aránega, S. (2017). La formación inicial de maestros y maestras a debate: ¿qué nos dicen sus protagonistas? Profesorado. Revista de Currículum y Formación del Profesorado, 21(1), 143-163.

Campamá, G. (2006). Gestión de los recursos humanos. La auditoría sociolaboral. Forum de Calidad, 176, 46-49.

Ciruela, A. (2009). La formación del capital humano como elemento de desarrollo de las cooperativas. Análisis de las necesidades formativas en las sociedades cooperativas mediante procesos de auditoría sociolaboral. CIRIEC-España. Revista de Economía Pública, Social y Cooperativa, 64, 85-104.

Dasgupta, P. and David, P. (1994). Towards a new economics of science. Research Policy, 23(5), 487-522.

Drejer, I. and Jørgensen, B.H. (2005). The dynamic creation of knowledge: analysing public private collaborations. Technovation, 25(2), 83-94.

Fuentes, F., Morales, A. y Veroz, R. (2005). Introducción a la auditoría sociolaboral. Murcia, España: Diego Marín, Librero-Editor.

Gibbons, M. et al. (1994). The New Production of Knowledge: The Dynamics of Science and Research in Contemporary Societies. Thousand Oaks, USA: SAGE.

George, D. and Mallery, P. (2003). SPSS/ PC+step by step: a simple guide and reference. Belmont, USA: Wadsworth Publishing Co.

Hall, B. (2003). On copyright and patent protection for software and databases: a tale of Two-worlds. En Granstrand, 0. (Ed.), Economics, Law and Intellectual Property (pp. 259-277). Tranbjerg, Denmark: Kluwer Academic Publishers. 
Hewitt-Dundas, N., Gkypalib, A. and Roperb, S. (2019). Does learning from prior collaboration help firms to overcome the 'two-worlds' paradox in university-business collaboration? Research Policy, 48(5), 1310-1322.

Huh, J., Delorme, D.E. and Reid, L.N. (2006). Perceived third-person effects and consumer attitudes on preventing and banning DTC advertising. Journal of Consumer Affairs, 40(1), 90-116.

Johnson, R.B., Onwuegbuzie, A.J. and Turner, L.A (2007). Toward a Definition of Mixed Methods Research. Journal of Mixed Methods Research, 1, 112-133.

Laursen, K. and Salter, A. (2004). Searching high and low: what types of firms use universities as a source of innovation? Research Policy, 33(8), 1201-1215.

Llanes, J., Figuera, P. y Torrado, M. (2017). Desarrollo de la empleabilidad y gestión personal de la carrera de graduados en pedagogía. Revista Española de Orientación y Psicopedagogía, 28(2), 46-60.

Mansfield, E. (1995). Academic research underlying industrial innovations - sources, characteristics and financing. The Review of Economics and Statistics, 77(1), 55-62.

Martensen, A. and Grønholdt, L. (2009). Quality in higher education: linking graduates' competencies and employers' needs. International Journal of Quality and Service Sciences, 1(1), 67-77.

McQuaid, R.W. and Lindsay, C. (2005). The concept of employability. Urban Studies, 42(2), 197-219.

Mulder, M. et al. (2009). The new competence concept in higher education: error or enrichment? Journal of European Industrial Training, 33(8-9), 755-770.

Méhaut, P. and Winch, C. (2012). The European qualification framework: skills, competences or knowledge? European Educational Research Journal, 11(3), 369-381.
Petruzzelli, A.M. (2011). The impact of technological relatedness, prior ties, and geographical distance on university-industry collaborations: a joint-patent analysis. Technovation, 31(7), 309-319.

Torras, E. y Ribas, J. (Coord.) (2005). Auditoria sociolaboral I. Barcelona, España: UOC.

van der Heijde, C.M. (2014). Employability and Self-Regulation in Contemporary Careers. En Coetzee, M. (Ed.), Psycho-social Career Metacapacities (pp. 7-17). London, England: Springer. 